\title{
Strengthening the case that elevated levels of programmed death ligand I predict poor prognosis in hepatocellular carcinoma patients
}

This article was published in the following Dove Press journal:

Journal of Hepatocellular Carcinoma

30 December 2016

Number of times this article has been viewed

\author{
Jian-Hong Zhongl,* \\ Cheng-Piao Luo ${ }^{2, *}$ \\ Chun-Yan Zhang ${ }^{2}$ \\ Le-Qun $\mathrm{Li}^{\prime}$
}

'Hepatobiliary Surgery Department, 2Experimental Department, Affiliated Tumor Hospital of Guangxi Medical University, Nanning, People's Republic of China

*These authors contributed equally to this work

Correspondence: Le-Qun Li Hepatobiliary Surgery Department, Affiliated Tumor Hospital of Guangxi Medical University, He Di Road, \#7I, Nanning 53002I, People's Republic of China

Tel +867715330855

Fax +86 77I 5312000

Email xitongpingjia@163.com

\begin{abstract}
Immunotherapy targeting programmed death receptor 1 and programmed death ligand 1 (PD-L1) has shown impressive antitumor efficacy in several solid cancers, including advanced hepatocellular carcinoma (HCC). Since response rates of various cancers to such immunotherapy appear to correlate with PD-L1 expression levels, several studies have examined whether PD-L1 expression correlates with HCC pathology and patient prognosis. In this paper, we analyzed the strength and limitations of a recent meta-analysis of associations of PD-L1 with HCC characteristics and patient prognosis.
\end{abstract}

Keywords: hepatocellular carcinoma, programmed death ligand 1, hepatic resection, prognoses

Hepatocellular carcinoma (HCC) is a malignant disease with poor prognosis. ${ }^{1}$ Its officially recommended treatment is by sorafenib therapy, which is extremely expensive, often causes adverse events, and prolongs overall survival by only 3 months in patients with advanced disease. ${ }^{2,3}$ Immunotherapy targeting programmed death receptor 1 (PD-1) and programmed death ligand 1 (PD-L1) has shown impressive antitumor efficacy in several solid cancers, ${ }^{46}$ including advanced HCC. ${ }^{7}$ Since response rates of various cancers to such immunotherapy appear to correlate with PD-L1 expression levels, ${ }^{8}$ several studies have examined whether PD-L1 expression correlates with HCC pathology and patient prognosis. As the results obtained were inconsistent, $\mathrm{Gu}$ et $\mathrm{al}^{9}$ initiated to perform the first meta-analysis that focused on the associations of PD-L1 with HCC characteristics and patient prognosis. ${ }^{9}$ They concluded that higher PD-L1 levels predict poor differentiation, vascular invasion, higher levels of $\alpha$-fetoprotein (AFP), and poorer survival. While these results are clinically useful, they should be interpreted with several limitations in mind.

One of the limitations is that the meta-analysis did not include four studies ${ }^{10-13}$ involving 384 patients that satisfied the inclusion criteria of this meta-analysis. ${ }^{9}$ In addition, one study ${ }^{14}$ included in this meta-analysis was based on PD-L1 assays in serum but not in tumor samples. The patients included in this study received both surgery and palliative therapies, while in other studies, ${ }^{15-20}$ patients received only surgery. These issues may increase heterogeneity in the pooled data, undermining the reliability of the results. In addition, it is unclear to us how this meta-analysis was able to report survival hazard ratios for the pooled patient population with tumors of any stage, when most studies in the meta-analysis reported survival separately by tumor stages but not in the population as a whole.

The work of Gu et al ${ }^{9}$ suggests that higher PD-L1 levels are associated with poorer clinicopathological characteristics of HCC. To extend this finding, we examined eleven 


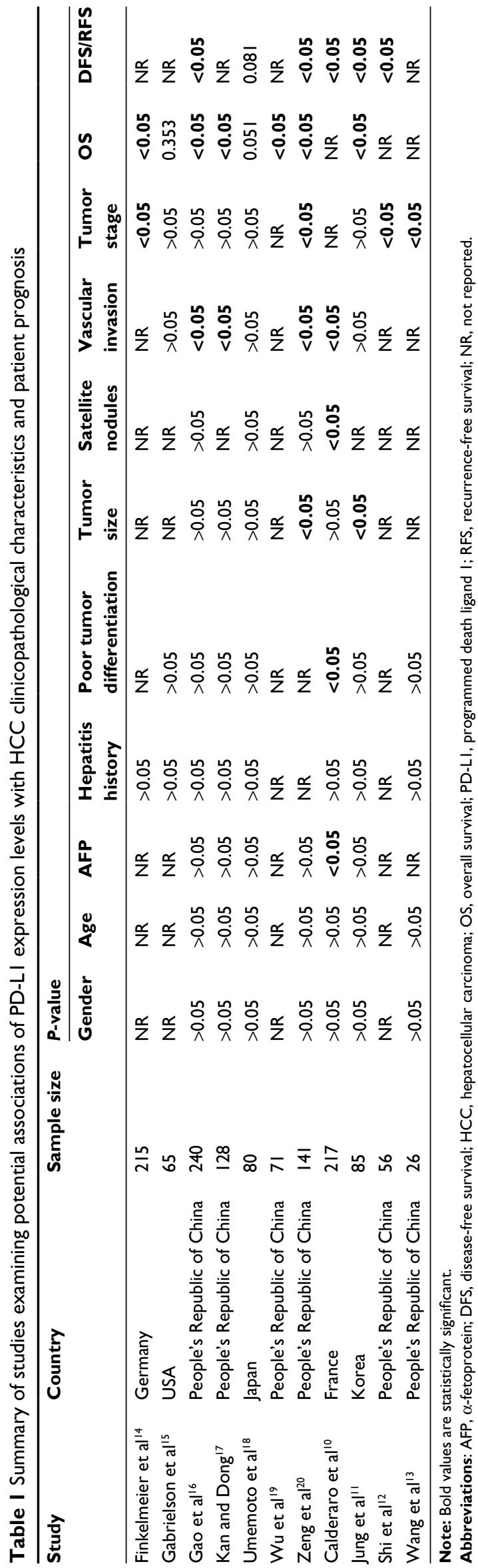

studies and found that none of the studies reported gender, age, or hepatitis history to be associated with elevated PD-L1 expression (Table 1). Only one study associated high PD-L1 expression with higher preoperative serum levels of AFP, poor tumor differentiation, and satellite nodules; ${ }^{10}$ two studies associated it with tumor size, ${ }^{11,20}$ four studies associated it with vascular invasion; $;^{10,16,17,20}$ and four studies ${ }^{12-14,20}$ associated it with tumor stage. One study reported no significant association between high PD-L1 levels and overall survival, ${ }^{15}$ while another study reported a nonsignificant trend that higher levels were associated with shorter overall survival. ${ }^{18}$

The results in Table 1 and those reported by $\mathrm{Gu}^{\mathrm{e}} \mathrm{al}^{9}$ suggest that elevated PD-L1 levels are associated with several HCC characteristics that are also risk factors for early tumor recurrence. Such recurrence can occur through two mechanisms: true metastasis due to primary HCC dissemination before surgery and multicentric occurrence (de novo) in remnant liver due to continuous viral infection and inflammation. ${ }^{21} \mathrm{HCC}$ treatments are usually effective against one or the other type of recurrence, but not both. In contrast, targeting PD-L1 may inhibit both types simultaneously, since reducing PD-L1 levels can strengthen T-cell responses to hepatitis virus infection. ${ }^{22,23}$

Despite its limitations, the meta-analysis of $\mathrm{Gu}$ et $\mathrm{al}^{9}$ substantially strengthens the evidence that higher PD-L1 levels are associated with poorer clinicopathological characteristics of HCC and poorer prognosis of patients. Further phase I or phase II clinical trials should be performed to investigate anti-PD-L1 treatment for HCC.

\section{Acknowledgments}

This work was funded by the National Natural Science Foundation of the People's Republic of China (81560460, 81060173), Guangxi University of Science and Technology Research Projects (KY2015LX056), the Self-Raised Scientific Research Fund of the Ministry of Health of Guangxi Province (Z2015621, Z2015601, GZZC15-34), and the Innovation Project of Guangxi Graduate Education (YCBZ2015030). The funding source had no role in the design or conduct of the study; in the collection, analysis, or interpretation of the data; or in the preparation, review, or approval of the manuscript.

\section{Disclosure}

The authors report no conflicts of interest in this work.

\section{References}

1. El-Serag HB. Hepatocellular carcinoma. N Engl J Med. 2011;365(12): 1118-1127. 
2. Zhong JH. The STORM trial and beyond: narrowing the horizon of adjuvant sorafenib for postoperative hepatocellular carcinoma. Tumour Biol. 2015;36(11):8271-8272.

3. Zhong JH, Du XK, Xiang BD, Li LQ. Adjuvant sorafenib in hepatocellular carcinoma: a cautionary comment of STORM trial. World $J$ Hepatol. 2016;8(23):957-960.

4. Robert C, Ribas A, Wolchok JD, et al. Anti-programmed-death-receptor-1 treatment with pembrolizumab in ipilimumab-refractory advanced melanoma: a randomised dose-comparison cohort of a phase 1 trial. Lancet. 2014;384(9948):1109-1117.

5. Brahmer JR, Tykodi SS, Chow LQ, et al. Safety and activity of antiPD-L1 antibody in patients with advanced cancer. $N$ Engl J Med. 2012;366(26):2455-2465.

6. Powles T, Eder JP, Fine GD, et al. MPDL3280A (anti-PD-L1) treatment leads to clinical activity in metastatic bladder cancer. Nature. 2014;515(7528):558-562.

7. El-Khoueiry AB, Melero I, Crocenzi T, et al. Phase I/II safety and antitumor activity of nivolumab in patients with advanced hepatocellular carcinoma (HCC). J Clin Oncol. 2015;33(Suppl) abstr LBA101.

8. Patel SP, Kurzrock R. PD-L1 Expression as a predictive biomarker in cancer immunotherapy. Mol Cancer Ther. 2015;14(4): 847-856.

9. Gu X, Gao XS, Xiong W, et al. Increased programmed death ligand-1 expression predicts poor prognosis in hepatocellular carcinoma patients. Onco Targets Ther. 2016;9:4805-4813.

10. Calderaro J, Rousseau B, Amaddeo G, et al. Programmed death ligand 1 expression in hepatocellular carcinoma: relationship with clinical and pathological features. Hepatology. 2016;64(6):2038-2046. doi: $10.1002 /$ hep. 28710 .

11. Jung HI, Jeong D, Ji S, et al. Overexpression of PD-L1 and PD-L2 is associated with poor prognosis in patients with hepatocellular carcinoma. Cancer Res Treat. 2016; doi: 10.4143/crt.2016.066.

12. Shi F, Shi M, Zeng Z, et al. PD-1 and PD-L1 upregulation promotes CD8(+) T-cell apoptosis and postoperative recurrence in hepatocellular carcinoma patients. Int J Cancer. 2011;128(4):887-896.
13. Wang BJ, Bao JJ, Wang JZ, et al. Immunostaining of PD-1/PD-Ls in liver tissues of patients with hepatitis and hepatocellular carcinoma. World J Gastroenterol. 2011;17(28):3322-3329.

14. Finkelmeier F, Canli O, Tal A, et al. High levels of the soluble programmed death-ligand (sPD-L1) identify hepatocellular carcinoma patients with a poor prognosis. Eur J Cancer. 2016;59:152-159.

15. Gabrielson A, Wu Y, Wang H, et al. Intratumoral CD3 and CD8 T-cell densities associated with relapse-free survival in HCC. Cancer Immunol Res. 2016;4(5):419-430.

16. Gao Q, Wang XY, Qiu SJ, et al. Overexpression of PD-L1 significantly associates with tumor aggressiveness and postoperative recurrence in human hepatocellular carcinoma. Clin Cancer Res. 2009;15(3):971-979.

17. Kan G, Dong W. The expression of PD-L1 APE1 and P53 in hepatocellular carcinoma and its relationship to clinical pathology. Eur Rev Med Pharmacol Sci. 2015;19(16):3063-3071.

18. Umemoto Y, Okano S, Matsumoto Y, et al. Prognostic impact of programmed cell death 1 ligand 1 expression in human leukocyte antigen class I-positive hepatocellular carcinoma after curative hepatectomy. J Gastroenterol. 2015;50(1):65-75.

19. Wu K, Kryczek I, Chen L, Zou W, Welling TH. Kupffer cell suppression of $\mathrm{CD} 8+\mathrm{T}$ cells in human hepatocellular carcinoma is mediated by B7-H1/programmed death-1 interactions. Cancer Res. 2009;69(20):8067-8075.

20. Zeng Z, Shi F, Zhou L, et al. Upregulation of circulating PD-L1/PD-1 is associated with poor post-cryoablation prognosis in patients with HBV-related hepatocellular carcinoma. PLoS One. 2011;6(9):e23621.

21. Zhong JH, Zhong QL, Li LQ, Li H. Adjuvant and chemopreventive therapies for resectable hepatocellular carcinoma: a literature review. Tumour Biol. 2014;35(10):9459-9468.

22. Grakoui A, John Wherry E, Hanson HL, Walker C, Ahmed R. Turning on the off switch: regulation of anti-viral $\mathrm{T}$ cell responses in the liver by the PD-1/PD-L1 pathway. J Hepatol. 2006;45(4):468-472.

23. Muhlbauer M, Fleck M, Schutz C, et al. PD-L1 is induced in hepatocytes by viral infection and by interferon-alpha and -gamma and mediates $\mathrm{T}$ cell apoptosis. J Hepatol. 2006;45(4):520-528.
Journal of Hepatocellular Carcinoma

\section{Publish your work in this journal}

The Journal of Hepatocellular Carcinoma is an international, peerreviewed, open access journal that offers a platform for the dissemination and study of clinical, translational and basic research findings in this rapidly developing field. Development in areas including, but not limited to, epidemiology, vaccination, hepatitis therapy, pathology and

\section{Dovepress}

molecular tumor classification and prognostication are all considered for publication. The manuscript management system is completely online and includes a very quick and fair peer-review system, which is all easy to use. Visit http://www.dovepress.com/testimonials.php to read real quotes from published authors. 